\title{
Une concertation locale
}

Les programmes de l'école danoise

\section{Peter Weng}

Traducteur : Patricia Vrinat

\section{OpenEdition}

\section{Journals}

Édition électronique

URL : http://journals.openedition.org/ries/3433

DOI : $10.4000 /$ ries.3433

ISSN : 2261-4265

\section{Éditeur}

Centre international d'études pédagogiques

\section{Édition imprimée}

Date de publication : 1 décembre 1996

Pagination : 105-112

ISSN : 1254-4590

\section{Référence électronique}

Peter Weng, « Une concertation locale », Revue internationale d'éducation de Sèvres [En ligne], 12 | 1996, mis en ligne le 01 octobre 2013, consulté le 30 avril 2019. URL : http://journals.openedition.org/ ries/3433 ; DOl : 10.4000/ries.3433 


\title{
Une concertation locale ${ }^{1}$
}

\section{Les programmes de l'école danoise}

\author{
Peter Weng
}

Au Danemark, en 1993, une nouvelle loi concernant la Folkeskole (école de base correspondant à l'enseignement primaire et aux premières années du secondaire) a été mise en application. Elle définit les objectifs et le cadre de travail dans ces écoles. Selon ce texte, le ministre de l'Éducation doit donner les directives touchant aux objectifs de l'enseignement, au contenu des cours et aux thèmes qu'il faut aborder dans les écoles. De plus, il peut donner des directives sur les programmes pour chaque matière ou thème de travail ainsi que des directives pour l'enseignement de ces matières ou de ces thèmes.

En 1994, selon les bases définies ci-dessus, le ministère de l'Éducation a fait publier un document intitulé "Les objectifs, les connaissances et les compétences de base " où sont définies les exigences du ministère de l'Éducation en ce qui concerne les objectifs, les savoirs minima et les compétences attendues dans les matières obligatoires et pour les thèmes à étudier.

Le contenu de ce document est le fruit du travail approfondi de sept commissions sur les programmes et de groupes de travail associés qui avaient été constitués pour chaque matière et chaque thème obligatoire dans les Folkeskolen. Les membres de ces commissions étaient des représentants des organisations des enseignants ou des parents ainsi que des personnes ayant des compétences particulières dans chaque matière ou sur le plan pédagogique. La coordination du travail des sept commissions a été effectuée par une autre commission comprenant des représentants, d'une part, des institutions éducatives qui prennent en charge les élèves à la fin de la Folkeskole et, d'autre part, des administrateurs locaux.

Le Conseil de la Folkeskole qui est indépendant des commissions a examiné le contenu final. Cet organisme, dont les membres sont nommés par le gouvernement, a une fonction de conseil. Les membres représentent les autorités administratives locales ainsi qu'une variété de groupes intéressés par l'éducation dans le primaire et dans les premières années du secondaire des écoles publiques. La structure du travail sur les programmes est présentée dans le tableau 1. Tout le processus de mise à jour des programmes des différentes matières et des thèmes obligatoires devrait être examiné dans la perspective des objectifs et du cadre établi par la loi sur la Folkeskole de 1993. Bien sûr, le contenu de cette loi s'appuie sur

1 Cet article a été traduit par Patricia Vrinat. 


\section{Tableau 1}

Organisation de la mise en place des programmes des matières et des thèmes de travail pour la Folkeskole

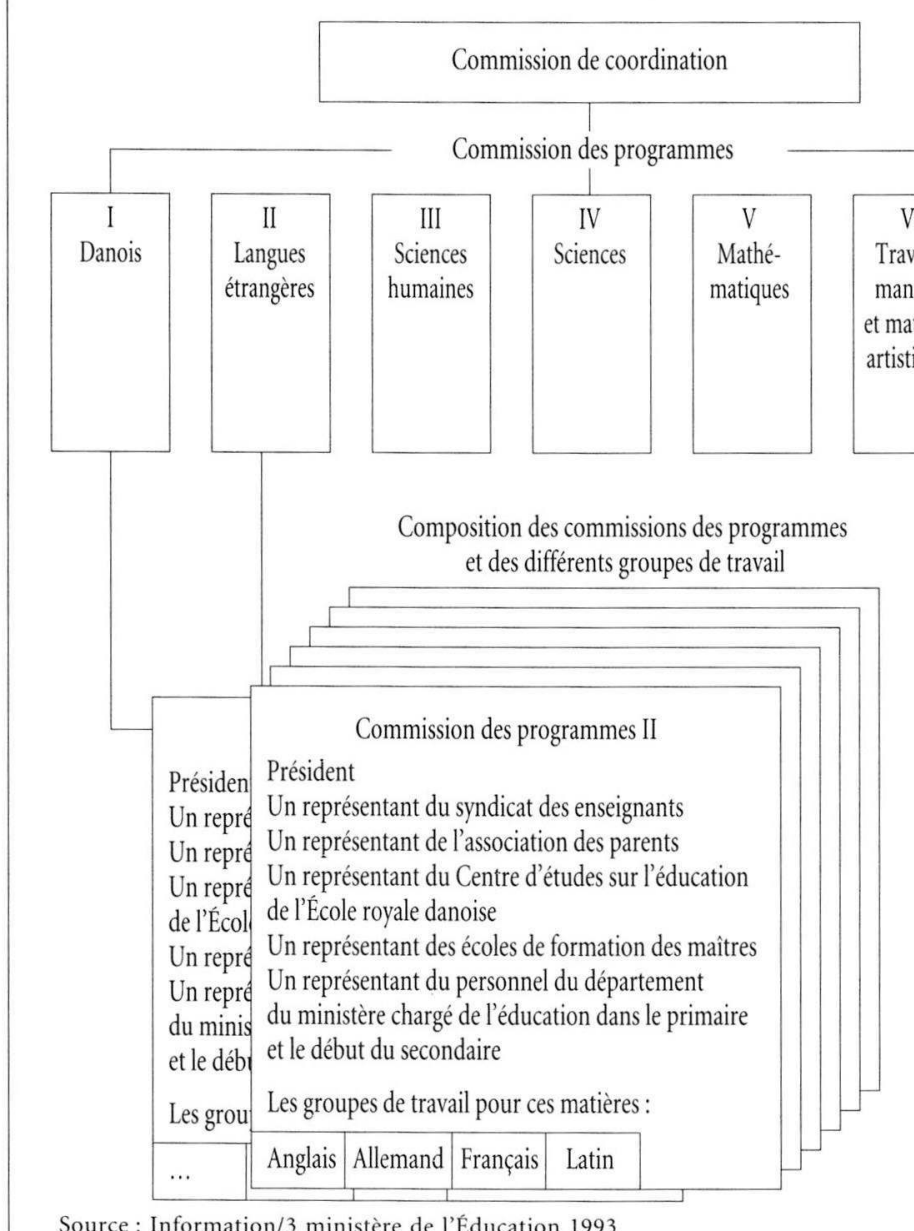

Source : Information/3 ministère de l'Éducation 1993

des lois antérieures, mais aussi sur une quantité de travail considérable et sur les innovations de plusieurs années.

Le Conseil de la Folkeskole, mentionné ci-dessus, a été créé en liaison avec la loi de 1993 et remplace le Conseil d'innovation de la Folkeskole qui fut à l'origine d'une grande partie du travail d'innovation qui sert de fondement aux nouveaux programmes. "Les objectifs, les connaissances et les compétences de base » est une publication assez directive en ce qui concerne les écoles et les autorités locales et c'est pour cela que les enseignants l'utilisent pour planifier leur enseignement et le mettre en place (voir p. 108 le descriptif de "L'éducation physique et sportive»). 
Comme cela a été mentionné auparavant, le ministre de l'Éducation a la possibilité de donner des directives sur les programmes et sur l'enseignement. Il faut insister sur la notion de possibilité car, d'après la loi sur la Folkeskole, il incombe aux municipalités de donner leur accord sur les programmes établis dans les différentes écoles de la région. Pour être exact, c'est au conseil d'administration de chaque école de mettre au point les programmes à soumettre au conseil municipal pour accord. Au conseil d'administration, les parents, les enseignants et les élèves sont représentés. Le président de ce conseil est toujours un représentant des parents et le directeur de l'école participe à toutes les réunions en tant que secrétaire. Il n'y a pas de règlements officiels sur la façon d'organiser le travail en ce qui concerne l'établissement du programme à l'intérieur de chaque école. Le directeur de l'école doit toutefois attirer l'attention du conseil sur les directives données par le ministre de l'Éducation et aussi sur les possibilités d'établir des programmes qui prennent en considération les attitudes et les facteurs d'une importance locale, tout en respectant le cadre de la loi sur la Folkeskole. Au début du préambule de la loi sur la Folkeskole, on trouve : "La Folkeskole devra promouvoir l'acquisition de connaissances et de compétences en coopération avec les parents... ". Ainsi, on insiste sur le fait que la coopération entre les parents et l'école est de toute première importance. De plus, la loi de 1993 souligne l'importance de donner aux élèves de l'expérience et des informations sur leur environnement local. La loi ouvre la voie à une meilleure coopération avec toute la communauté locale telles d'autres institutions, associations et la vie économique locale, pour que l'école devienne un centre culturel local avec un sens plus large que dans le passé.

C'est sur ces bases et en donnant des informations au conseil de l'école sur des attitudes pédagogiques proposées, que le directeur peut prendre des initiatives et faire adopter, par le conseil, des programmes à soumettre à la municipalité, qui peuvent s'écarter des directives ministérielles ou bien les suivre. Ensuite, les programmes acceptés par le conseil municipal deviennent obligatoires pour les enseignants.

Bien que la loi implique que les écoles conçoivent leurs propres programmes, pratiquement toutes les écoles soumettent les directives ministérielles aux conseils municipaux pour approbation. Pour le moment, il n'y a pas de statistiques sur la fréquence de programmes locaux. Même si on est loin de l'établissement local de programmes par rapport aux possibilités offertes par la loi sur la Folkeskole, le développement local d'une école passe par une réflexion sur des programmes locaux. Dans ce but, le ministère de l'Éducation a préparé des matériaux informatifs qui donnent des conseils généraux et pratiques pour ce travail.

Les directives de travail, édictées par la loi de 1993 et le document «Les objectifs, les connaissances et les compétences de base", doivent être suivies obligatoirement par les enseignants et les autorités locales. Dans la préface des 


\section{L'ÉDUCATION PHYSIQUE ET SPORTIVE}

L'enseignement de l'éducation physique et sportive aura les objectifs, les compétences et les connaissances suivants :

\section{Les objectifs}

L'objectif de l'enseignement de l'éducation physique et sportive est que les élèves, par leur expérience de sports de tout genre et leur réflexion sur ces sports, arrivent à avoir les compétences et les savoirs qui les aident dans leur développement physique et général.

Les élèves devront avoir la possibilité de ressentir la joie et le désir de participer à un sport, et de développer tous les prérequis qui leur feront comprendre l'importance des activités physiques tout au long de leur vie, en liaison avec la nature, la culture et la société dans laquelle ils vivent.

Les élèves devront avoir un aperçu et une certaine expérience des conditions nécessaires à la bonne santé et la culture physique. L'enseignement devrait les amener à se prendre en charge et à coopérer avec d'autres.

Les connaissances et les compétences de base

L'enseignement de l'éducation physique et sportive concernera une variété d'activités et doit prendre en compte l'aspect ludique, compétitif, expressif et expérimental. Il y aura également des aspects physiques, psychologiques, sociaux et culturels.

En faisant le lien entre la théorie et la pratique, les élèves peuvent acquérir une compréhension des liens entre la découverte, la compétence et le savoir et relier perception et savoir.

Il est de première importance que les élèves travaillent aussi bien avec des enseignants que des enseignantes, et qu'ils participent à la fois à des activités mixtes ou non.

Des formes variées de mouvements

L'enseignement comportera des formes variées de mouvements et des connaissances sur le corps et le sport. Les élèves travailleront à partir de compétences physiques de base et apprendront les différents types d'action à effectuer pour mettre en pratique le sport. Ils s'engageront complètement dans le travail et auront la possibilité de créer, de s'évaluer, de s'entrainer, de travailler en groupe et de participer à des compétitions.

\section{L'expérience personnelle}

L'enseignement ira de pair avec les réactions propres des élèves et leur façon de se situer dans le monde qui les entoure. Ils apprendront comment le corps réagit à des situations différentes et ils tiendront compte des réactions et des sentiments qui surviennent quand ils agissent avec d'autres. L'enseignement tiendra compte des impressions et expressions physiques, ainsi que de la communication et de l'expérience du corps dans le développement et l'apprentissage de chaque élève.

\section{Les conditions physiques et biologiques}

On fera en sorte que les élèves aient des connaissances sur des conditions physiques et biologiques et sachent quelles sont les meilleures pratiques pour l'entraînement.

\section{Les valeurs éthiques et esthétiques}

Les valeurs éthiques et esthétiques feront partie de l'enseignement et on insistera sur les aspects esthétiques. Les élèves apprendront le lien entre la nature, le corps et la société, et cela leur servira de base pour comprendre et apprendre l'interaction entre la santé, les conditions de vie et la façon de vivre.

\section{Culture physique}

Le point de départ de l'enseignement de l'éducation physique sera la tradition sportive danoise, et les élèves auront la possibilité d'acquérir de l'expérience par la variété de cette culture. La culture physique d'autres pays sera incluse pour faire en sorte que des élèves fassent des comparaisons. 
directives sur les programmes du ministère, le ministre écrit : "Quand il s'agit d'établir des programmes locaux - ou d'opérer des changements par rapport aux directives du ministre -, il faut souligner le fait que les programmes locaux doivent être écrits de façon à ce qu'un bon nombre d'éléments des directives ministérielles restent clairement parmi les priorités. »

Les programmes doivent donner un cadre aux connaissances et aux compétences nécessaires pour que les élèves développent leur personnalité au cours de leur scolarité et deviennent des citoyens responsables. Donc, les programmes ne devraient pas être des descriptifs isolés pour des matières distinctes, mais replacés dans un ensemble qui permette à l'élève de développer ses connaissances dans plusieurs domaines. L'objectif n'est pas de créer des spécialistes de différentes matières, mais de donner aux élèves une base pour toutes les expériences de la vie. On peut y arriver par l'interdisciplinarité et par un travail par projets, comme cela est souligné dans la nouvelle loi sur la Folkeskole.

Il s'ensuit que trois éléments devraient être inclus dans tous les programmes, en conformité avec la nouvelle loi. La technologie de l'information se développe à une vitesse telle qu'elle doit être incluse comme outil dans l'enseignement d'un maximum de matières. Comme l'interaction de l'homme avec la nature est de première importance pour les générations futures, il devrait $\mathrm{y}$ avoir une "touche verte» dans toutes les matières à chaque fois qu'il est possible de le faire d'une manière naturelle dans le contexte. Ce n'est pas seulement dans les matières scientifiques que l'on devrait aborder l'environnement et d'autres questions importantes liées à la nature. Dans toutes les matières, on devrait examiner des questions d'éthique générale sur la nature ainsi que sur l'environnement, pour que l'élève ait la possibilité de se forger une opinion sur l'attitude et l'action à avoir à chaque fois qu'une interaction avec la nature est concernée. Le dernier élément qu'il faudrait inclure dans toutes les matières est la dimension pratique et créative. Les programmes ne devraient pas se limiter à des indications sur le contenu, mais devraient aussi indiquer différentes façons de travailler et de s'exprimer. Dans toutes les matières, y compris celles qui ont la réputation d'être très théoriques, on doit s'attacher à donner plus d'importance à cette dimension et on doit offrir davantage de possibilités de travailler d'une manière pratique. On doit créer des situations qui permettent aux élèves d'appréhender les matières avec tous leurs sens plus souvent que dans le passé.

L'extrait suivant des directives ministérielles pour les mathématiques constitue un exemple de présentation du contenu du programme et montre la façon dont les trois éléments mentionnés ci-dessus pourraient y être incorporés. Les programmes peuvent être divisés en séquences ou selon le niveau des classes, surtout pour les mathématiques. On trouve le descriptif des programmes pour trois niveaux : le niveau débutant (classes 1 à 3), le niveau intermédiaire (classes 3 à 7) et le niveau supérieur (classe 7 à 9). Au-delà, à partir de la classe 10, 


\section{DIRECTIVES MINISTÉRIELLES POUR LES MATHÉMATIQUES}

\section{Extrait du niveau débutant}

"L'enseignement se fonde sur tous les prérequis que les élèves possèdent lorsqu'ils débutent à l'école. Les élèves utilisent les nombres dans leur vie de tous les jours. Ils ont l'expérience de décrire des objets et des événements par le dessin, et ils sont capables de comprendre des informations avec quelques expressions mathématiques.

Les élèves y ajoutent différentes expériences de cette matière par le jeu, des activités et des investigations à l'école et dans son environnement. La compréhension intuitive des mathématiques que les élèves utilisent au départ se développe et devient une conceptualisation mathématique.

Dans leur travail, les élèves utilisent différentes formes d'expression qui incluent le corps, les sens et la langue.

En ce qui concerne les jeux et les expériences, les élèves peuvent acquérir une expérience de départ par le hasard. Dans tous les domaines de cette matière la résolution de problèmes est un élément important, surtout quand il s'agit d'expériences et d'investigations. Il se peut que les élèves aient affaire à des questions du genre : "Que se passe-t-il si...? Est-ce que cela pourrait être ainsi parce que...?»

Dans certains cas l'ordinateur peut être un moyen d'expérimentation. »

\section{Extrait du niveau intermédiaire}

"Au niveau intermédiaire, il est important que les élèves prennent confiance et croient que cette matière leur permettra d'avoir un outil universel pour résoudre des problèmes pratiques et théoriques. Par la concertation, les élèves doivent se rendre compte de l'importance de fournir des efforts multiples pour appréhender la matière. L'expérience quotidienne, ainsi que celle acquise à l'école, est toujours le point de départ de l'enseignement. Les élèves développent une compréhension des mathématiques et de leurs origines par leur propre implication dans l'établissement de concepts mathématiques.

La résolution de problèmes et les méthodes de travail.

Par tous les aspects de la matière abordés dans leur travail, les élèves devraient avoir l'occasion d'acquérir des méthodes de travail comme, par exemple, la formulation de problèmes, l'investigation et la description de règles.

Pour la formulation de problèmes et l'investigation, les élèves travaillent en avançant des hypothèses qui, à ce niveau, se limitent à un caractère de « devinettes et de vérifications ».

La formulation de problèmes et la réflexion font partie de cette activité et permettent de savoir comment les mathématiques peuvent être employées pour répondre aux questions formulées. Les investigations peuvent aussi bien concerner l'aspect appliqué de la matière que les problèmes formulés pour illustrer les concepts mathématiques.

Il peut s'agir de travailler dans un environnement bien défini en utilisant du matériel concret, par exemple en travaillant avec un «tableau à clous ", ou en utilisant des logiciels sur un ordinateur.

Un dialogue sur les concepts mathématiques et les activités forme une partie centrale de l'enseignement. Les élèves formulent des règles à partir de leurs expériences et des intuitions qui en découlent. "

\section{Extrait du niveau supérieur}

"A ce niveau les élèves sont plus à même de planifier seuls leurs propres activités et de s'impliquer dans les domaines à étudier et les tâches à faire. Ils sont capables, seuls et avec l'aide des autres, d'acquérir des connaissances mathématiques nouvelles, et ils peuvent travailler à de nouvelles applications des mathématiques. 
Le calcul et le dessin peuvent se faire à l'aide d'une calculette ou d'un ordinateur. C'est ainsi que l'on peut se concentrer sur le travail de différents chapitres à étudier et les problèmes associés, mais en montrant que les mathématiques peuvent être appliquées à des problèmes réels. Des exemples choisis montreront les applications possibles des mathématiques comme outil pour résoudre certains problèmes de société dans le domaine de l'économie, de la technologie et de l'environnement.

Les élèves doivent apprendre à utiliser et à interpréter des statistiques comme celles qui sont employées par les médias et d'autres sources d'information. Ils doivent travailler sur l'influence des choix dans la façon de communiquer les résultats et l'influence que cela peut avoir dans la compréhension des données réelles.

L'application des modèles mathématiques permet aux élèves de simplifier le problème en question dans le modèle. Il sera également possible de voir quelles sont les valeurs qui se cachent derrière le choix d'un certain modèle mathématique. "

l'enseignement est décrit séparément, ce niveau n'étant pas obligatoire mais laissé au choix des élèves.

La nouvelle loi sur la Folkeskole définit un système qui permet de placer l'élève au cœur de l'enseignement. Ainsi l'enseignant doit fonder son enseignement sur l'expérience acquise par l'élève à son arrivée à l'école. Les extraits cités soulignent cette approche ainsi que l'importance d'une pédagogie différenciée et l'interdisciplinarité. Les programmes doivent couvrir le terrain le plus large possible, pour que l'enseignant puisse planifier son travail de façon à ce que l'élève soit mis au défi d'incorporer les connaissances acquises dans d'autres matières ou en dehors de l'école.

Les écoles qui conçoivent leurs propres programmes utiliseront probablement les directives ministérielles comme modèle pour mettre en place leurs projets locaux. En chargeant ou en modifiant ce modèle il est possible, dans une certaine mesure, d'imprimer la marque de l'école sur le programme, tout en respectant les règles imposées par la nouvelle loi et par le ministère.

On devrait mentionner le fait que la loi sur la Folkeskole indique que "l'organisation de l'enseignement, y compris le choix des enseignements et des méthodes de travail, du matériel pédagogique et le choix du contenu, doit, dans tous les cas, arriver à la hauteur des objectifs de la Folkeskole et cet enseignement doit être varié pour prendre en compte les besoins et prérequis de chaque élève. " Cette section stipule que les programmes devraient être décrits d'une manière très globale, pour pouvoir prendre en considération les différences entre les élèves. Ceci signifie que l'enseignant peut travailler dans un cadre très large en ce qui concerne le contenu choisi comme base pour l'enseignement et les méthodes à employer. Cependant, cela ne peut être réalisé sans prise en compte des élèves qui sont impliqués par la loi dans leur propre éducation comme indiqué dans l'extrait suivant: «Au niveau de chaque classe et dans chaque matière, l'enseignant et l'élève travailleront ensemble pour faire en sorte 
que les objectifs soient atteints. L'organisation du travail de l'élève tiendra compte de ces objectifs. La mise en place de méthodes de travail et le choix du contenu se feront, dans la mesure du possible, par une consultation entre l'élève et l'enseignant. » Donc, le contenu des programmes peut faire l'objet d'un choix. Pour être significatif, il faut que les programmes locaux soient rédigés de façon à ce que non seulement le conseil d'administration mais aussi l'élève puisse comprendre.

Les élèves peuvent choisir de terminer l'étude de toutes ces matières par un examen et les programmes locaux, ainsi que l'enseignement qui les accompagne, doivent répondre aux exigences des examens de fin d'étude de la Folkeskole. C'est la responsabilité du directeur de faire en sorte que l'école atteigne ses objectifs et que les programmes soient approuvés par la municipalité.

La source principale d'information de l'enseignant sur les programmes ne consiste pas dans les directives du ministère, mais dans les trente « brochures par matières " publiées par le ministère pour les différentes disciplines et thèmes d'étude obligatoires. Elles doivent en priorité aider à l'enseignement en décrivant tout ce qui est particulièrement pertinent. Chaque petit livret donne les objectifs de la matière, les connaissances de base et les compétences demandées, le contenu du programme, un guide pour l'enseignement et, si la matière peut aboutir à un examen, les exigences de cet examen.

De plus, le livret donne des conseils sur le matériel pédagogique et une description de la responsabilité de l'enseignant par rapport aux objectifs et à l'évaluation de la matière. Les paragraphes les plus importants des livrets concernent la pédagogie et les séquences d'enseignement. Une illustration de ce dernier point est "l'environnement dans l'enseignement des mathématiques" où la « touche verte " peut être incorporée dans l'enseignement selon les intentions prévues pour l'ensemble des matières.

Ces livrets par matière sont écrits par la commission sur les programmes déjà mentionnée. Cela peut être un avantage, puisqu'il garantit une certaine continuité dans le travail sur les différentes matières depuis la législation jusqu'au guide de l'enseignant. Cependant cela peut aussi imposer des limites dans les réflexions didactiques exprimées dans les programmes et dans les guides. Il est également assez fréquent que les membres des commissions sur les programmes soient aussi des auteurs de manuels; il y a alors souvent un lien très proche entre les guides et les manuels pour une matière donnée. Il peut en résulter, dans ce cas de figure, que l'enseignement soit influencé par les manuels qui servent à la fois de programmes et de guides, ce qui va à l'encontre des intentions de la nouvelle loi sur la Folkeskole. 